\title{
Prise de position de la Société Francophone du Diabète (SFD) sur la prise en charge médicamenteuse de l'hyperglycémie du patient diabétique de type 2
}

\author{
Management of hyperglycemia in type 2 diabetes: \\ Position Statement of the Francophone Diabetes Society
}

Patrice Darmon, Bernard Bauduceau, Lyse Bordier, Jacques Bringer, Gérard Chabrier, Bernard Charbonnel, Bruno Detournay, Pierre Fontaine, André Grimaldi, Pierre Gourdy, Bruno Guerci, Alfred Penfornis, Jean-Pierre Riveline, André Scheen, pour la Société Francophone du Diabète (SFD).

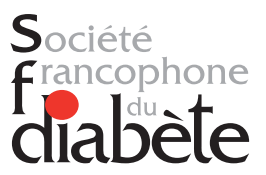

\author{
Composition du groupe de travail de \\ la SFD \\ Pr Bernard Bauduceau (endocrino- \\ logue-diabétologue, Saint-Mandé), \\ Pr Lyse Bordier (endocrinologue-dia- \\ bétologue, Saint-Mandé), Pr Jacques \\ Bringer (endocrinologue-diabétologue, \\ Montpellier), Dr Gérard Chabrier (endo- \\ crinologue-diabétologue, Strasbourg), \\ Pr Bernard Charbonnel (endocrino- \\ logue-diabétologue, Nantes), Pr Patrice \\ Darmon (endocrinologue-diabéto- \\ logue, Marseille), Dr Bruno Detournay \\ (CEMKA-EVAL, Bourg-la-Reine), $\mathrm{Pr}$ \\ Pierre Fontaine (endocrinologue-dia- \\ bétologue, Lille), Pr André Grimaldi \\ (endocrinologue-diabétologue, Paris), \\ Pr Pierre Gourdy (endocrinologue-dia- \\ bétologue, Toulouse), Pr Bruno Guerci \\ (endocrinologue-diabétologue, Nancy), \\ Pr Alfred Penfornis (endocrinologue- \\ diabétologue, Corbeil-Essonnes), $\mathrm{Pr}$ \\ Jean-Pierre Riveline (endocrinologue- \\ diabétologue, Paris), Pr André Scheen \\ (endocrinologue-diabétologue, Liège)
}

\author{
Coordination de la rédaction \\ Pr Patrice Darmon (Marseille)
}

\begin{abstract}
Composition du groupe de relecture Pr Brigitte Dormont, Professeur d'Économie (Paris I)

Pr Jean Doucet, interniste gériatre et diabétologue, Professeur de Thérapeutique (Rouen)

Pr Serge Halimi, endocrinologue-diabétologue, Professeur Emérite de Nutrition (Grenoble)

Pr Anne-Marie Magnier, Professeur de Médecine Générale (Paris VI)

Dr Sylvie Picard, endocrinologue-diabétologue (Dijon)

M. Gérard Raymond, Président de la Fédération Française des Diabétiques

Dr Dominique Simon, docteur en Santé Publique (ICAN, Paris)

Dr Pierre Sérusclat, endocrinologue-diabétologue (Vénissieux)
\end{abstract}

\section{Correspondance}

Société Francophone du Diabète (SFD) Secrétariat Permanent 60 rue Saint-Lazare

75009 Paris

www.sfdiabete.org

\section{Les membres du groupe de relecture ont émis un certain nombre de remarques et de suggestions sur ce texte. Certaines d'entre elles ont été prises en compte par le groupe de travail de la SFD, d'autres non. Cependant, tous les relecteurs susnommés ont accepté d'endosser la prise de position de la SFD dans la version finale présentée ici.}




\section{Conflits d'intérêt des membres du groupe de travail}

- Bernard Bauduceau déclare avoir participé à des interventions ponctuelles (essais cliniques, travaux scientifiques, activité de conseil, conférence ou colloque) pour les entreprises AstraZeneca, Eli Lilly, Merck Sharp \& Dohme (MSD), Novartis, Novo Nordisk, Sanofi, Vitalaire.

- Lyse Bordier déclare avoir participé à des interventions ponctuelles (essais cliniques, travaux scientifiques, activité de conseil, conférence ou colloque) pour les entreprises AstraZeneca, Becton Dickinson (BD), Boehringer Ingelheim, Bristol-Myers Squibb, Eli Lilly, Jansen, Merck Sharp \& Dohme (MSD), Novartis, Novo Nordisk, Sanofi.

- Jacques Bringer déclare avoir participé à des formations organisées par l'industrie pharmaceutique et à des rencontres d'experts avec les laboratoires Lilly France, Merck Sharp \& Dohme (MSD), Novo Nordisk, Sanofi.

- Gérard Chabrier déclare avoir participé à des interventions ponctuelles (travaux scientifiques, activité de conseil, conférence ou colloque) et avoir été pris en charge pour participer à des congrès pour/par les entreprises Eli Lilly, Ipsen, LVL Médical, Merck-Serono, Novartis, Novo Nordisk, Pfizer, Roche Diagnostics, Sanofi, Vitalaire.

- Bernard Charbonnel déclare avoir touché des honoraires pour des activités de conseil ou des conférences pour les entreprises AstraZeneca, Boehringer Ingelheim, Eli Lilly, Merck Sharp \& Dohme (MSD), Novo Nordisk, Sanofi, Takeda.

- Patrice Darmon déclare avoir participé à des interventions ponctuelles (essais cliniques, travaux scientifiques, activité de conseil, conférence ou colloque) pour les entreprises AstraZeneca, Bastide Médical, Bayer, Boehringer Ingelheim, Eli Lilly, LVL Médical, Merck Sharp \& Dohme (MSD), Novartis, Novo Nordisk, Sanofi.

- Bruno Detournay est employé de CEMKA-EVAL, un bureau d'études fournissant des prestations d'études et de conseils pour l'ensemble des acteurs privés et publics dans le champ de la santé. II a reçu des honoraires pour des activités de conseil ou des conférences pour les entreprises Merck Sharp \& Dohme (MSD), Novo Nordisk, Sanofi.

- Pierre Fontaine déclare avoir participé à des interventions ponctuelles (essais cliniques, travaux scientifiques, activité de conseil, conférence ou colloque) pour les entreprises Abbott, AstraZeneca, Becton Dickinson (BD), Eli Lilly, Merck Sharp \& Dohme (MSD), Novartis, Novo Nordisk, Sanofi.

- André Grimaldi déclare avoir reçu des honoraires pour des activités de conseil ou des conférences pour les entreprises Abbott, Amgen, Eli Lilly, Merck Sharp \& Dohme (MSD), Sanofi, Vitalaire.

- Pierre Gourdy déclare avoir reçu des honoraires occasionnels, à titre personnel ou institutionnel, pour des activités d'orateur, de conseiller scientifique ou de recherche clinique, de la part des laboratoires AstraZeneca, Boehringer Ingelheim, BristolMyers Squibb, Eli Lilly, GlaxoSmithKline, Janssen, Merck Sharp \& Dohme (MSD), Novartis, Novo Nordisk, Sanofi, Servier, Takeda.

- Bruno Guerci déclare avoir participé à des interventions ponctuelles (essais cliniques, travaux scientifiques, activité de conseil, conférence ou colloque) pour les entreprises Abbott, AstraZeneca, Boehringer Ingelheim, Bristol-Myers Squibb, Dinno Santé, Eli Lilly, GlaxoSmithKline, Intarcia, Janssen Pharmaceutica, Johnson \& Johnson, Medtronic, Menarini Diagnostics, Merck Sharp \& Dohme (MSD), Metacure, Novartis, Novo Nordisk, Orkyn', Pfizer, Roche Diagnostics, Sanofi, Vitalaire.

- Alfred Penfornis déclare avoir participé à des interventions ponctuelles (essais cliniques, travaux scientifiques, activité de conseil, conférence ou colloque) pour les entreprises AstraZeneca, Eli Lilly, Medtronic, Merck Sharp \& Dohme (MSD), Novartis, Novo Nordisk, Sanofi.

- Jean-Pierre Riveline déclare avoir participé à des interventions ponctuelles (activité de conseil, conférence ou colloque) pour les entreprises Abbott, Eli Lilly, Johnson \& Johnson, Novo Nordisk, Sanofi.

- André Scheen déclare avoir participé à des interventions ponctuelles (essais cliniques, travaux scientifiques, activité de conseil, conférence ou colloque) pour les entreprises AstraZeneca, Boehringer Ingelheim, Eli Lilly, Janssen Pharmaceutica, Merck Sharp \& Dohme (MSD), Novartis, Novo Nordisk, Sanofi.

\section{Conflits d'intérêt des membres du groupe de relecture}

- Brigitte Dormont déclare ne pas avoir de conflit d'intérêt en lien avec cet article.

- Jean Doucet déclare avoir participé à des interventions ponctuelles (conférences, rédaction de documents pédagogiques) pour les entreprises Becton Dickinson (BD), Eli Lilly, Nestlé HomeCare, Novo Nordisk, Sanofi. Il est promoteur d'une étude épidémiologique partiellement subventionnée par la Fondation Novo Nordisk et Merck-Serono.

- Serge Halimi déclare avoir participé à des interventions ponctuelles (essais cliniques, travaux scientifiques, activité de conseil, conférence ou colloque) pour les entreprises AstraZeneca, Boehringer Ingelheim, Eli Lilly, Janssen Pharmaceutica, LifeScan, Merck Sharp \& Dohme (MSD), Novartis, Novo Nordisk, Sanofi.

- Anne-Marie Magnier déclare ne pas avoir de conflit d'intérêt en lien avec cet article.

- Sylvie Picard déclare avoir participé à des interventions ponctuelles (essais cliniques, travaux scientifiques, activités de conseil, conférences ou colloques) pour les entreprises Abbott, Animas, AstraZeneca, Bayer Diagnostics, Eli Lilly, Janssen Pharmaceutica, LifeScan, Medtronic, Merck Sharp \& Dohme (MSD), Novartis, Novo Nordisk, Pierre Fabre, Sanofi, Takeda, Vitalaire.

- Gérard Raymond déclare ne pas avoir de conflit d'intérêt en lien avec cet article.

- Dominique Simon déclare avoir exercé des activités de conseil pour le laboratoire Sanofi.

- Pierre Sérusclat déclare avoir participé à des interventions ponctuelles (essais cliniques, travaux scientifiques, activités de conseil, conférences ou colloques) pour les entreprises Abbott, Eli Lilly, Johnson \& Johnson, Medtronic, Merck Sharp \& Dohme (MSD), Novartis, Novo Nordisk, Roche, Sanofi, Takeda, Vitalaire. 
Prise de position de la Société Francophone du Diabète (SFD) sur la prise en charge médicamenteuse de l'hyperglycémie du patient diabétique de type 2

Patrice Darmon, Bernard Bauduceau, Lyse Bordier, Jacques Bringer, Gérard Chabrier, Bernard Charbonnel, Bruno Detournay, Pierre Fontaine, André Grimaldi, Pierre Gourdy, Bruno Guerci, Alfred Penfornis, Jean-Pierre Riveline, André Scheen, pour la Société Francophone du Diabète (SFD).

\section{Sommaire}

A. Bénéfice de l'équilibre glycémique sur la micro- et la macroangiopathie

B. Médecine fondée sur les preuves et décision médicale partagée

C. Individualisation des objectifs glycémiques

D. Réévaluation de la réponse thérapeutique et règles d'arrêt des traitements

E. Stratégie thérapeutique dans le diabète de type 2 (situation « commune »)

1. Au moment de la découverte du diabète

2. Si $\mathrm{HbA}_{1 \mathrm{c}}>$ objectif personnalisé malgré les modifications thérapeutiques du mode de vie

3. Si $\mathrm{HbA}_{1 \mathrm{c}}>$ objectif personnalisé malgré modifications thérapeutiques du mode de vie et metformine à dose maximale tolérée bien observée

4. Si $\mathrm{HbA}_{1 \mathrm{c}}>$ objectif personnalisé malgré modifications thérapeutiques du mode de vie + bithérapie à dose optimale bien observée
a. Après une bithérapie metformine + iDPP4
b. Après une bithérapie metformine + sulfamide hypoglycémiant
c. Après une bithérapie metformine + GLP-1 RA

5. Si $\mathrm{HbA}_{1 \mathrm{c}}>$ objectif personnalisé malgré modifications thérapeutiques du mode de vie + trithérapie à dose optimale bien observée (hors insuline)

6. Initiation d'une insulinothérapie basale

7. Si $\mathrm{HbA}_{1 \mathrm{c}}>$ objectif personnalisé malgré modifications thérapeutiques du mode de vie + insuline basale bien titrée et bien observée

8. En cas d'intolérance avérée ou de contre-indication à la metformine

\section{F. Populations particulières}

1. Patient âgé de plus de $\mathbf{7 5}$ ans

2. Patient obèse avec indice de masse corporelle (IMC) $\geq 35 \mathrm{~kg} / \mathrm{m}^{2}$

3. Patient présentant une insuffisance rénale chronique (IRC)

4. Patient en prévention cardiovasculaire secondaire

5. Patient présentant une insuffisance cardiaque

6. Patiente enceinte ou envisageant de l'être

G. Place de l'auto-surveillance glycémique chez le patient diabétique de type 2

\section{H. Dimension économique}

\section{Prise de position de la Société Francophone du Diabète (SFD) sur la prise en charge médicamenteuse de l'hyperglycémie du patient diabétique de type 2}

- Compte tenu des évolutions majeures survenues ces dernières années dans le domaine du diabète de type 2 (DT2), il est apparu nécessaire à la Société Francophone du Diabète (SFD) de missionner un groupe de travail afin de rédiger une prise de position sur la prise en charge médicamenteuse du DT2, prenant en compte les nouvelles données. L'objectif de cette prise de position est de clarifier une démarche devenue complexe et de personnaliser les parcours thérapeutiques selon les situations cliniques et le rapport bénéfices/risques, sans oublier les aspects pharmaco-économiques de la prise en charge globale. Cette prise de position s'appuie sur les principes bien compris de la médecine fondée sur les preuves (evidence-based medicine), prenant en compte ses trois composantes - les preuves scientifiques, l'expertise du clinicien, et la préférence des patients - pour la mise en œuvre d'une médecine personnalisée et centrée sur le patient.

- Le groupe de travail a fait le choix de ne pas attribuer de grade aux propositions qui seront faites dans ce document car ces propositions sont, dans la majorité des cas, fondées sur des avis d'experts pour ce qui relève des stratégies thérapeutiques. - La SFD s'engage à réactualiser la prise de position sur la prise en charge du DT2 tous les deux ans de manière à pouvoir la faire évoluer au gré des dernières connaissances scientifiques et de l'accessibilité éventuelle à de nouveaux médicaments. - La version longue de cette prise de position (argumentaire, références bibliographiques) est disponible sur le site de la SFD (www.sfdiabete.org). 


\section{A. Bénéfice de l'équilibre glycémique sur la micro- et la macroangiopathie}

Avis $\mathrm{n}^{\circ} 1$ - Bénéfice de l'équilibre glycémique sur la micro- et la macroangiopathie

- La prévention des complications du DT2 exige une prise en charge de l'ensemble des facteurs de risque, passant obligatoirement par un contrôle optimisé de l'équilibre glycémique. - Le bénéfice d'un équilibre glycémique optimal sur les complications micro-vasculaires est largement démontré. Ce bénéfice existe également pour les complications macrovasculaires (notamment les infarctus du myocarde), mais ne devient significatif qu'après un temps plus prolongé de suivi.

- Un critère de substitution est un critère intermédiaire capable de prédire la survenue d'événements cliniques. Au regard de la littérature scientifique disponible, I'hémoglobine glyquée $\left(\mathrm{HbA}_{1 \mathrm{c}}\right)$ peut être considérée comme un critère de substitution acceptable pour la survenue des complications micro-vasculaires du diabète, mais pas pour celle des complications macro-vasculaires.

\section{B. Médecine fondée sur les preuves et décision médicale partagée}

\section{Avis $n^{\circ} 2$ - Médecine fondée sur les preuves} et décision médicale partagée

- La médecine fondée sur les preuves vise à prendre les meilleures décisions médicales personnalisées pour chaque patient et repose sur les connaissances scientifiques établies, mais aussi sur l'expertise et l'expérience du clinicien, le profil du patient, ses préférences et ses choix.

- L'approche centrée sur le patient implique une décision médicale partagée, fondée sur l'échange d'informations détaillées autour de toutes les options possibles et conclue par une prise de décision éclairée, acceptée mutuellement par le patient et le soignant.

\section{Individualisation des objectifs glycémiques}

Avis $n^{\circ} 3$ - Individualisation des objectifs glycémiques (tableau I)

- L'objectif d'HbA $\mathrm{H}_{1 \mathrm{c}}$ doit être individualisé selon le profil du patient et co-décidé avec lui, et peut donc évoluer au fil du temps.

- Pour la plupart des patients diabétiques de type 2, une cible d' $\mathrm{HbA}_{1 \mathrm{c}}$ inférieure ou égale à $7 \%(53 \mathrm{mmol} / \mathrm{mol})$ est recommandée.
- Pour les patients diabétiques de type 2 dont le diabète est nouvellement diagnostiqué ET dont l'espérance de vie est supérieure à 15 ans ET sans antécédent cardiovasculaire, une cible d' $\mathrm{HbA}_{1 \mathrm{c}}$ inférieure ou égale à $6,5 \%$ (48 $\left.\mathrm{mmol} / \mathrm{mol}\right)$ est recommandée, sous réserve d'être atteinte par la mise en œuvre ou le renforcement des modifications thérapeutiques du mode de vie puis, si cela est insuffisant, par un ou plusieurs traitements ne provoquant pas d'hypoglycémie.

- Une cible d' $\mathrm{HbA}_{1 \mathrm{c}}$ inférieure ou égale à $8 \%(64 \mathrm{mmol} / \mathrm{mol})$ pourra être proposée chez d'autres patients, en fonction de l'âge, de l'espérance de vie, de l'ancienneté du diabète, du risque d'hypoglycémie, ainsi que des co-morbidités associées, notamment rénales et cardiovasculaires (cf Avis $\mathrm{n}^{\circ} 23$ et 25$)$; une cible d' $\mathrm{HbA}_{1 \mathrm{c}}$ inférieure ou égale à $9 \%(75 \mathrm{mmol} /$ mol) sera proposée chez le sujet âgé dépendant et/ou à la santé très altérée (voir Avis $\mathrm{n}^{\circ} 20$ ).

\section{Réévaluation de la réponse thérapeutique et règles d'arrêt des traitements}

Avis $n^{\circ} 4$ - Réévaluation de la réponse

thérapeutique et règles d'arrêt

- L'efficacité thérapeutique et la tolérance de tout antihyperglycémiant devront être réévaluées 3 à 6 mois après son introduction - voire plus rapidement en cas de signes cliniques liés à l'hyperglycémie, de la survenue d'hypoglycémies ou d'une intolérance au traitement.

- Au moment de réévaluer la réponse thérapeutique, il convient de porter une attention particulière à l'adhésion du patient au traitement et de lutter contre toute inertie médicale, que ce soit pour arrêter un médicament insuffisamment efficace ou, à l'inverse, pour intensifier la stratégie de traitement si besoin.

- Les sulfamides, les glinides, les inhibiteurs de la dipeptidyl peptidase 4 (iDPP4) et les agonistes des récepteurs du glucagon-like peptide-1 (GLP-1 RA) seront arrêtés si la baisse d' $\mathrm{HbA}_{1 \mathrm{c}}$ est de moins de $0,5 \%$ (et que l' $\mathrm{HbA}_{1 \mathrm{c}}$ reste supérieure à l'objectif) 3 à 6 mois après l'initiation du traitement, à condition que la titration ait été adéquate (sulfamides, glinides, certains GLP-1 RA), que l'adhésion au traitement soit jugée satisfaisante et en l'absence de facteur identifié de déséquilibre glycémique.

- Sous sulfamides et sous glinides, une attention particulière devra être portée au risque hypoglycémique et ces agents seront arrêtés en cas d'hypoglycémies répétées ou sévères.

- La réévaluation de la réponse thérapeutique et les règles d'arrêt permettent d'éviter un « empilement » thérapeutique systématique au fil des années chez le patient diabétique de type 2. 
Tableau I. Objectifs d'HbA $1 \mathrm{c}$ selon le profil du patient.

\begin{tabular}{|c|c|c|}
\hline & Profil du patient & $\mathrm{HbA}_{1 \mathrm{c}}$ cible \\
\hline \multirow[t]{3}{*}{ Cas général } & La plupart des patients avec un DT2 & $\leq 7 \%$ \\
\hline & $\begin{array}{l}\text { DT2 nouvellement diagnostiqué, dont l'espérance de vie est } \\
>15 \text { ans et sans antécédent cardiovasculaire }\end{array}$ & $\leq 6,5 \%{ }^{1}$ \\
\hline & $\begin{array}{l}\text { DT2: } \\
\text { - avec une comorbidité grave avérée et/ou une espérance de } \\
\text { vie limitée ( }<5 \text { ans) } \\
\text { - ou avec des complications macro-vasculaires évoluées } \\
\text { - ou ayant une longue durée d'évolution du diabète ( }>10 \text { ans) } \\
\text { et pour lesquels la cible de } 7 \% \text { s'avère difficile à atteindre car } \\
\text { l'intensification thérapeutique provoque des hypoglycémies } \\
\text { sévères }\end{array}$ & $\leq 8 \%$ \\
\hline \multirow[t]{3}{*}{ Personnes âgées² } & $\begin{array}{l}\text { Dites « en bonne santé », bien intégrées socialement et } \\
\text { autonomes d'un point de vue décisionnel et fonctionnel, et dont } \\
\text { l'espérance de vie est jugée satisfaisante }\end{array}$ & $\leq 7 \%$ \\
\hline & $\begin{array}{l}\text { Dites « fragiles » à l'état de santé intermédiaire et à risque de } \\
\text { basculer dans la catégorie des « dépendants et/ou à la santé } \\
\text { très altérée " }\end{array}$ & $\leq 8 \%$ \\
\hline & $\begin{array}{l}\text { Dites « dépendantes et/ou à la santé très altérée ", en raison } \\
\text { d'une polypathologie chronique évoluée génératrice de } \\
\text { handicaps et d'un isolement social }\end{array}$ & $\begin{array}{l}<9 \% \\
\text { et/ou glycémies capillaires } \\
\text { préprandiales entre } 1 \text { et } 2 \mathrm{~g} / \mathrm{L}\end{array}$ \\
\hline \multirow[t]{2}{*}{$\begin{array}{l}\text { Patients avec antécédents } \\
\text { (ATCD) cardiovasculaires }\end{array}$} & $\begin{array}{l}\text { ATCD de maladie cardiovasculaire considérée comme non } \\
\text { évoluée }\end{array}$ & $\leq 7 \%$ \\
\hline & ATCD de maladie cardiovasculaire considérée comme évoluée ${ }^{3}$ & $\leq 8 \%$ \\
\hline \multirow{2}{*}{$\begin{array}{l}\text { Patients avec insuffisance rénale } \\
\text { chronique (IRC) }\end{array}$} & IRC modérée (stades 3A et 3B) & $\leq 7 \%$ \\
\hline & IRC sévère et terminale (stade 4 et 5 ) & $\leq 8 \%$ \\
\hline \multirow{2}{*}{$\begin{array}{l}\text { Patientes enceintes ou } \\
\text { envisageant de l'être } \\
\text { (diabète préexistant à la } \\
\text { grossesse) }\end{array}$} & Avant d'envisager la grossesse & $\leq 6,5 \%$ \\
\hline & Durant la grossesse & $\begin{array}{c}\leq 6,5 \% \\
\text { et/ou } \\
\text { glycémies }<0,95 \mathrm{~g} / \mathrm{L} \text { à jeun et }<1,20 \mathrm{~g} / \mathrm{L} \\
\text { en postprandial à } 2 \text { heures }\end{array}$ \\
\hline
\end{tabular}

${ }^{1}$ Sous réserve d'être atteint par la mise en œuvre ou le renforcement des modifications thérapeutiques du mode de vie puis, en cas d'échec, par un ou plusieurs traitements ne provoquant pas d'hypoglycémie.

${ }^{2}$ De manière générale, chez les sujets âgés, il est essentiel de minimiser le risque d'hypoglycémie, notamment d'hypoglycémie sévère. Ce risque existe sous sulfamides hypoglycémiants (« sulfamides " dans ce texte), répaglinide, et insuline, et il est plus important lorsque l'HbA est inférieure à 7 \%.

${ }^{3}$ Infarctus du myocarde (IDM) avec insuffisance cardiaque, atteinte coronarienne sévère (atteinte du tronc commun ou atteinte tritronculaire ou atteinte de l'artère interventriculaire antérieure proximale), atteinte polyartérielle (au moins deux territoires artériels symptomatiques), artériopathie oblitérante des membres inférieurs symptomatique, accident vasculaire cérébral récent $(<6$ mois).

${ }^{4}$ Stades $3 A$ : débit de filtration glomérulaire (DFG) entre 45 et $59 \mathrm{~mL} / \mathrm{min} / 1,73 \mathrm{~m}^{2}$; $3 \mathrm{~B}$ : DFG entre 30 et $44 \mathrm{~mL} / \mathrm{min} / 1,73 \mathrm{~m}{ }^{2} ;$ stade $4:$ DFG entre 15 et $29 \mathrm{~mL}$ $\mathrm{min} / 1,73 \mathrm{~m}^{2} ;$ stade $5: \mathrm{DFG}<15 \mathrm{~mL} / \mathrm{min} / 1,73 \mathrm{~m}^{2}$.

\section{E. Stratégie thérapeutique dans le diabète de type 2 (situation “ commune ")}

- Dans les chapitres suivants, sont abordées différentes situations cliniques dans lesquelles sont présentées les alternatives thérapeutiques possibles, fondées sur les données de la littérature et l'expérience des experts du groupe de travail de la SFD. La prise en compte de la troisième composante de la médecine fondée sur les preuves, les préférences du patient, consiste à présenter les avantages et inconvénients de chacune de ces alternatives thérapeutiques et à en discuter avec le patient. Les cliniciens peuvent s'appuyer, pour ce faire, sur des outils d'aide à la décision, tel que le tableau /l présenté ci-dessous. - Dans tous les cas, la mise en œuvre de modifications thérapeutique du mode de vie, la participation et l'adhésion du patient au traitement devront être réévaluées avant tout changement et/ou toute intensification thérapeutique, dont les modalités devront, en outre, être co-décidées avec le patient. Tout changement et/ ou toute intensification thérapeutique doit être couplée à une éducation thérapeutique et à un accompagnement du patient. 


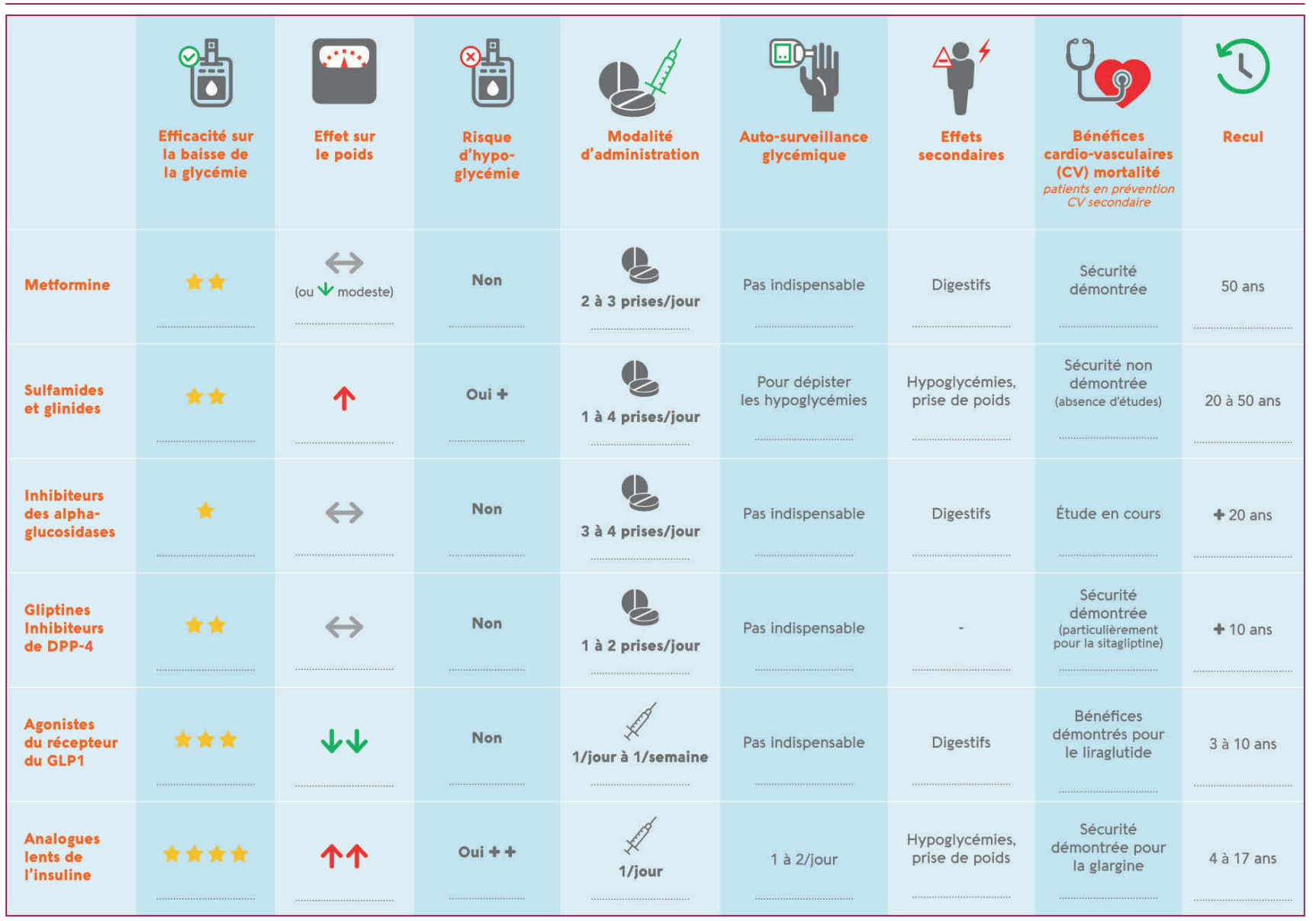

Avis $n^{\circ} 5$ - Participation et adhésion thérapeutique du patient

- La mise en œuvre de modifications thérapeutiques du mode de vie (changement des habitudes alimentaires, lutte contre la sédentarité, activité physique adaptée), la participation et l'adhésion du patient au traitement devront être réévaluées avant tout changement et/ou toute intensification thérapeutique, dont les modalités devront, en outre, être co-décidées avec le patient.

- Tout changement et/ou toute intensification thérapeutique doit être couplée à une éducation thérapeutique et à un accompagnement du patient.

\section{Au moment de la découverte du diabète}

Avis $n^{\circ} 6$ - Au moment du diagnostic de diabète : modifications thérapeutiques du mode de vie

- Au moment du diagnostic, il est indispensable de proposer des modifications thérapeutiques du mode de vie (changement des habitudes alimentaires, lutte contre la sédentarité, activité physique adaptée) et l'effet de ces mesures doit être évalué au bout de 3 à 6 mois avant de proposer une thérapeutique médicamenteuse - la metformine, sauf contre-indication ou intolérance vraie - si l'HbA $\mathrm{Ac}_{1 \mathrm{c}}$ reste supérieure à l'objectif.

- Si l'on estime, d'un commun accord avec le patient, que les modifications thérapeutiques du mode de vie ne suffiront pas pour atteindre l'objectif d' $\mathrm{HbA}_{1 c}$, un traitement médicamenteux - la metformine, sauf contre-indication ou intolérance avérée - peut être proposé d'emblée.

- Les changements des habitudes alimentaires et d'activité physique doivent, à chaque fois que possible, donner lieu à un accord avec le patient sur des objectifs spécifiques, réalistes, mesurables, temporellement déterminés. 
Avis $\mathrm{n}^{\circ} 7$ - Au moment du diagnostic

de diabète : cas particuliers

- Au moment du diagnostic, on pourra proposer une bithérapie d'emblée en cas de déséquilibre glycémique initial important $\left(\mathrm{HbA}_{1 \mathrm{c}}>9 \%\right.$ ou $\left.75 \mathrm{mmol} / \mathrm{mol}\right)$.

- Une insulinothérapie peut être indiquée d'emblée en cas de déséquilibre glycémique majeur $\left(\mathrm{HbA}_{1 \mathrm{c}}>10 \%\right.$ ou $86 \mathrm{mmol} /$ $\mathrm{mol}$ ), en particulier en présence d'un syndrome polyuropolydipsique et/ou d'une perte de poids involontaire, et est indispensable en cas d'hyperglycémie majeure avec hyperosmolarité ou en présence de corps cétoniques (cétonurie ou cétonémie positive). Dans ces cas-là, le recours à l'insulinothérapie peut être transitoire et un relais par d'autres médicaments anti-hyperglycémiants peut être envisagé secondairement - sauf dans certaines situations particulières, et notamment lorsque ce tableau clinique révèle en fait un diabète de type 1 .

\section{Si HbA $\mathrm{H}_{1 \mathrm{c}}>$ objectif personnalisé malgré les} modifications thérapeutiques du mode de vie

Avis $\mathrm{n}^{\circ} 8$ - Objectif d’ $\mathrm{HbA}_{1 \mathrm{c}}$ non atteint malgré les modifications thérapeutiques du mode de vie

Lorsque l'objectif d'HbA $\mathrm{Ac}_{1 \mathrm{c}}$ 'est pas atteint malgré les modifications thérapeutiques du mode de vie, on proposera en première intention un traitement par metformine, à doses progressives jusqu'à la dose maximale tolérée (idéalement entre 2 et $3 \mathrm{~g} / \mathrm{jour}$ ), fractionnée en deux ou trois prises.

\section{Si HbA ${ }_{1 c}>$ objectif personnalisé}

malgré modifications thérapeutiques

du mode de vie et metformine à dose maximale tolérée bien observée

Avis $\mathrm{n}^{\circ} 9$ - Objectif d’ $\mathrm{HbA}_{1 \mathrm{c}}$ non atteint sous metformine (figure 1 )

- L'association metformine + iDPP4 doit être préférée à une bithérapie metformine + sulfamide lorsque l'objectif d' $\mathrm{HbA}_{1 \mathrm{c}}$ n'est pas atteint sous metformine en monothérapie en raison d'un haut niveau de preuve en faveur des iDPP4 sur l'absence de risque hypoglycémique et la sécurité cardiovasculaire ; en outre, la sitagliptine a démontré une absence de risque vis-à-vis de l'insuffisance cardiaque chez des patients en prévention cardiovasculaire secondaire (voir Avis $\left.\mathrm{n}^{\circ} 26\right)$. À efficacité égale sur l' $\mathrm{HbA}_{1 \mathrm{c}}$, il y a un avantage médical à choisir un iDPP4 plutôt qu'un sulfamide.
- L'association metformine + sulfamide expose à un risque d'hypoglycémie et de prise de poids. Elle est moins onéreuse que la bithérapie metformine + iDPP4, mais elle nécessite une auto-surveillance glycémique. Elle peut être proposée à des patients à faible risque hypoglycémique.

- L'association metformine + GLP-1 RA peut être envisagée lorsque l'objectif d' $\mathrm{HbA}_{1 \mathrm{c}}$ n'est pas atteint sous metformine :

- chez le patient obèse (indice de masse corporelle, IMC $\geq$ $30 \mathrm{~kg} / \mathrm{m}^{2}$ ), tout en tenant compte du prix élevé des GLP-1 RA; pour des raisons économiques, il convient de privilégier le GLP-1 RA dont le coût journalier est le moins élevé ;

- chez le patient en situation de prévention cardiovasculaire secondaire, en choisissant alors le liraglutide, compte tenu des bénéfices démontrés dans l'étude LEADER (pour Liraglutide Effect and Action in Diabetes: Evaluation of cardiovascular outcomes Results) (voir Avis $\mathrm{n}^{\circ} 26$ ).

- L'efficacité des iDPP4 et des GLP-1 RA devra être soigneusement réévaluée à intervalles réguliers, compte tenu de leur prix supérieur à celui des sulfamides.

4. Si $\mathrm{HbA}_{1 \mathrm{c}}>$ objectif personnalisé malgré modifications thérapeutiques du mode de vie + bithérapie à dose optimale bien observée

\section{a. Après une bithérapie metformine + iDPP4}

Avis $\mathrm{n}^{\circ} 10$ - Objectif d’'HbA ${ }_{1 c}$ non atteint sous bithérapie metformine + iDPP4 (figure 2)

- Lorsque l'objectif d' $\mathrm{HbA}_{1 \mathrm{c}}$ n'est pas atteint sous bithérapie metformine + iDPP4 :

- une possibilité est de passer à une trithérapie orale metformine + iDPP4 + sulfamide, ce qui permet de surseoir à la prescription d'un traitement injectable ;

- une autre possibilité est d'arrêter l'iDPP4 et de passer à une association metformine + GLP-1 RA, plus efficace sur l'HbA1c et sur le poids. Le choix du liraglutide sera privilégié chez le patient en prévention cardiovasculaire secondaire (voir Avis $\left.n^{\circ} 26\right)$; sinon, il conviendra de privilégier le GLP-1 RA dont le coût journalier est le moins élevé ; - une troisième possibilité est d'instaurer une insulinothérapie basale en association à la metformine (voir Avis $n^{\circ} 17$ pour la gestion des anti-hyperglycémiants lors de l'instauration d'une insuline basale).

- Dans le cas particulier d'une réponse thérapeutique insuffisante 3 ou 6 mois après initiation de l'iDPP4 (voir Avis $n^{\circ} 4$ ), une bithérapie alternative metformine + sulfamide pourra également être envisagée. 


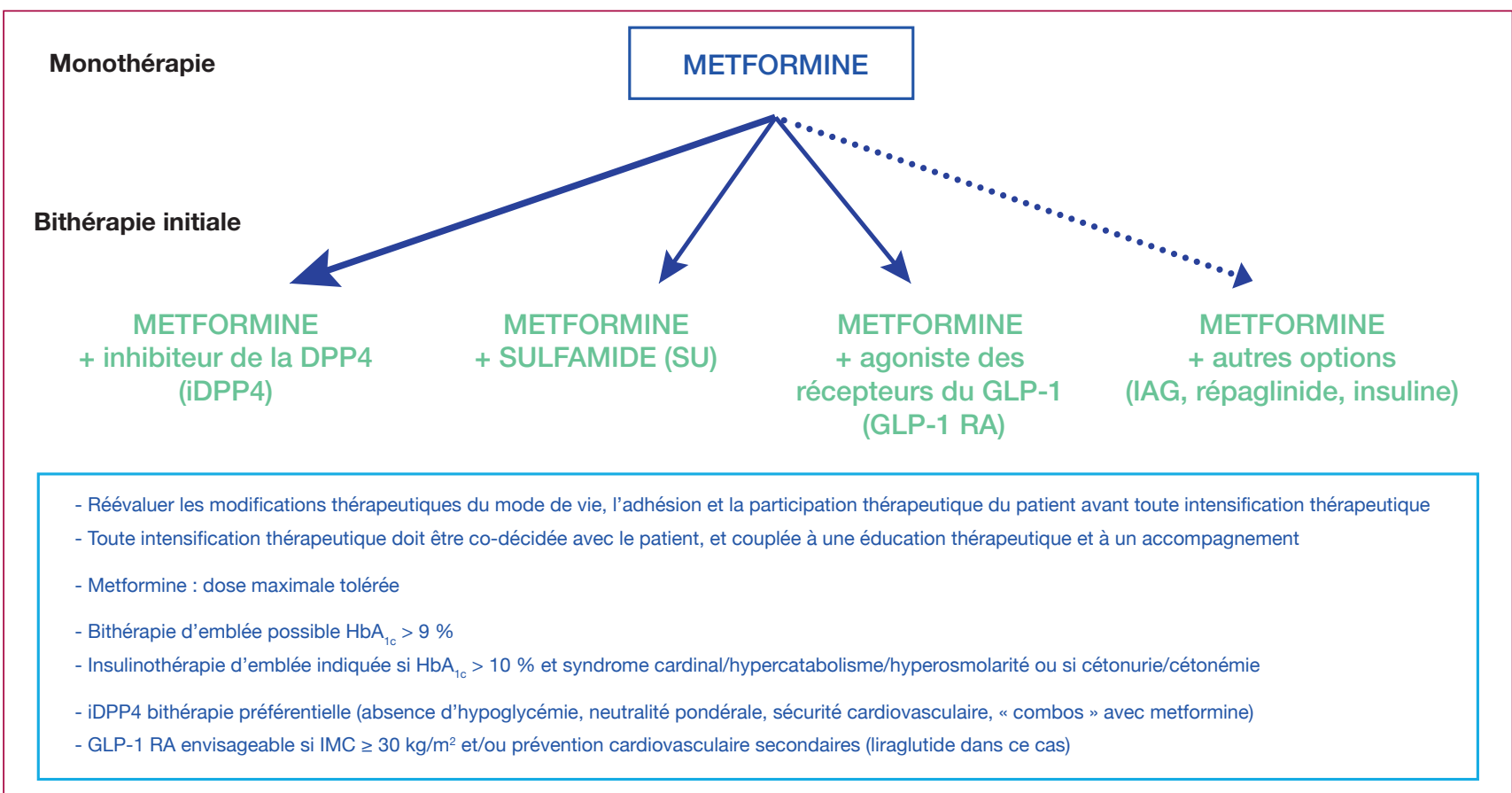

Figure 1. Stratégie thérapeutique si $\mathrm{HbA}_{1 \mathrm{c}}>$ objectif personnalisé malgré modifications thérapeutiques du mode de vie et monothérapie par metformine à dose maximale tolérée bien observée.

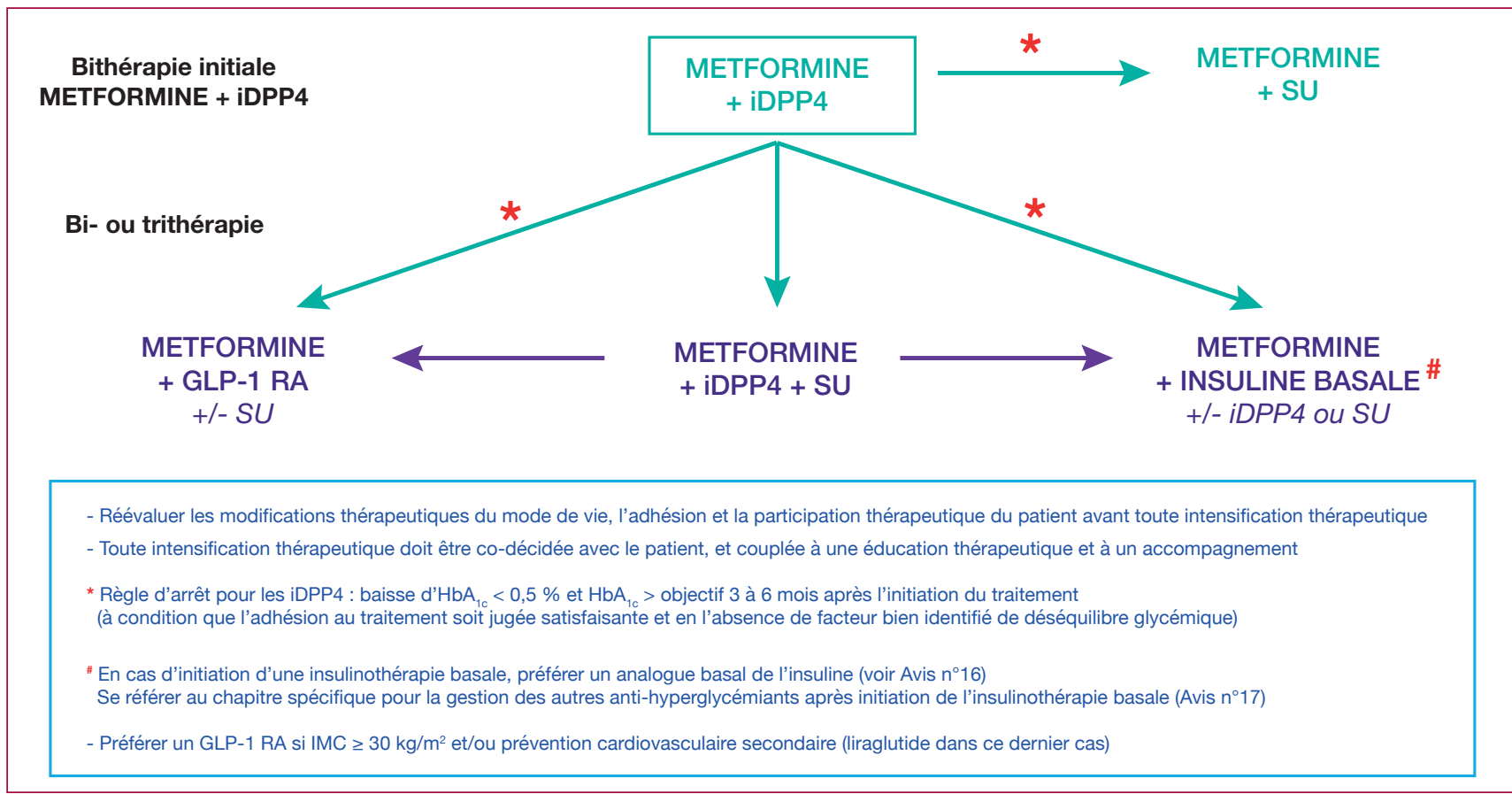

Figure 2. Stratégie thérapeutique si $\mathrm{HbA}_{1 \mathrm{c}}>$ objectif personnalisé malgré modifications thérapeutiques du mode de vie + bithérapie metformine + iDPP4 à dose optimale bien observée. 


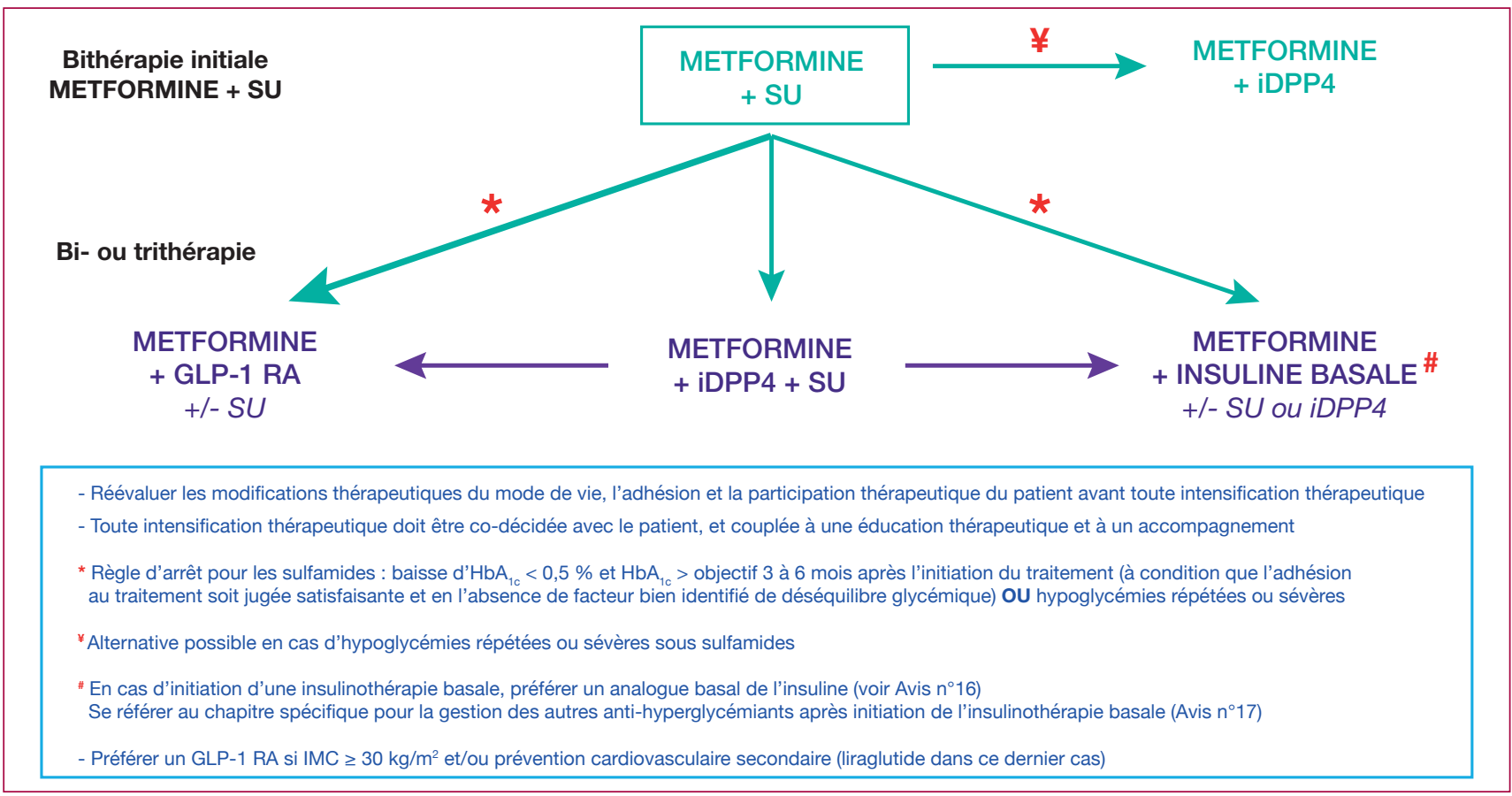

Figure 3. Stratégie thérapeutique si $\mathrm{HbA}_{1 \mathrm{c}}>$ objectif personnalisé malgré modifications thérapeutiques du mode de vie + bithérapie metformine + sulfamide à dose optimale bien observée.

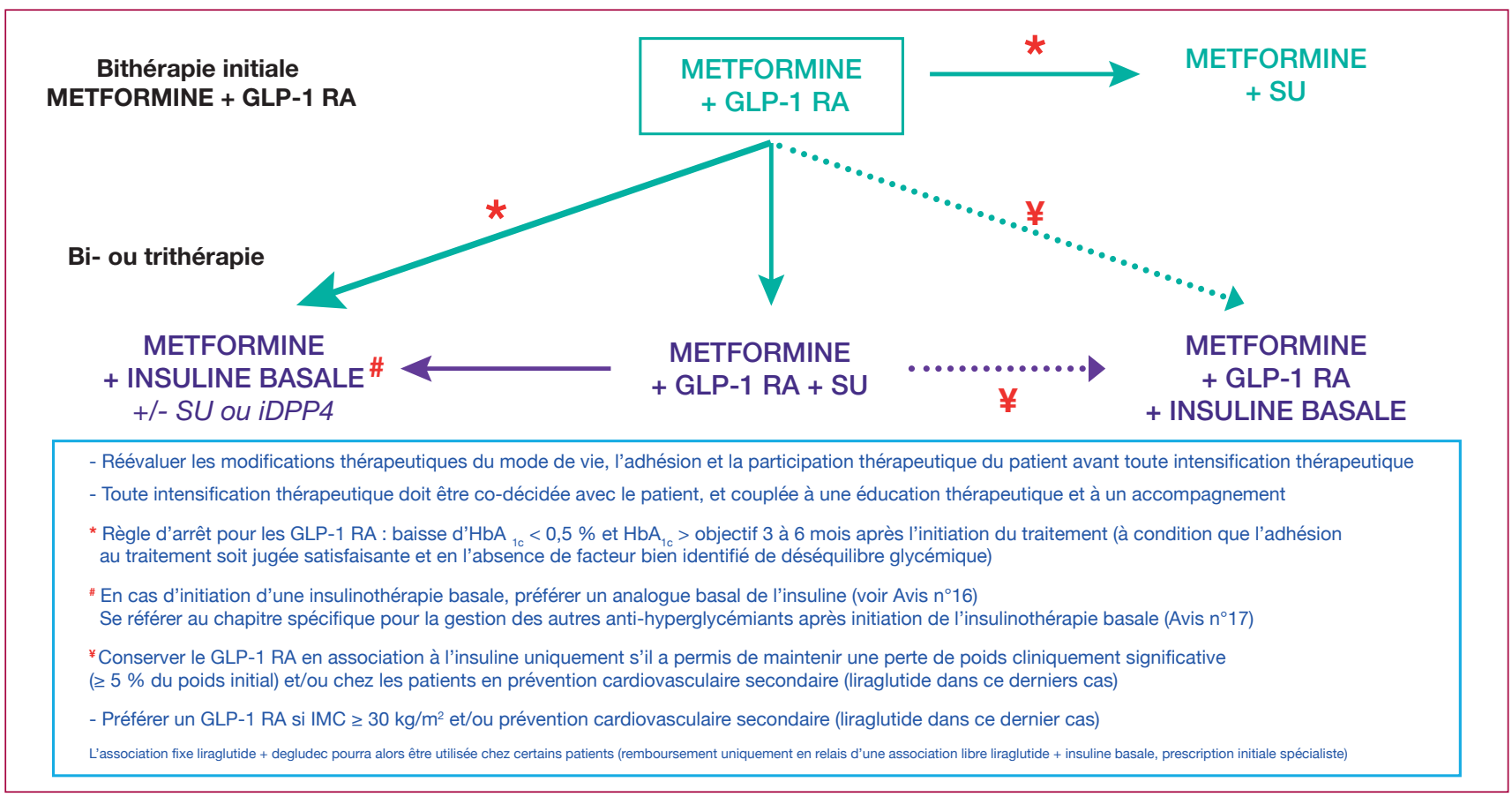

Figure 4. Stratégie thérapeutique si $\mathrm{HbA}_{1 \mathrm{c}}>$ objectif personnalisé malgré modifications thérapeutiques du mode de vie + bithérapie metformine + GLP-1 RA à dose optimale bien observée. 


\section{b. Après une bithérapie metformine + sulfamide hypoglycémiant}

Avis $\mathrm{n}^{\circ} 11$ - Objectif d’ $\mathrm{HbA}_{1 \mathrm{c}}$ non atteint sous bithérapie metformine + sulfamide hypoglycémiant (figure 3 )

- Lorsque l'objectif d' $\mathrm{HbA}_{1 \mathrm{c}}$ n'est pas atteint sous bithérapie metformine + sulfamide :

- une possibilité est de passer à une trithérapie metformine + sulfamide + iDPP4, qui permet de surseoir à la prescription d'un traitement injectable, mais est moins efficace sur l'HbA1c que les deux autres alternatives ;

- une autre possibilité est d'ajouter un GLP-1 RA, avec le choix pour le clinicien d'arrêter ou de conserver le sulfamide, en baissant sa posologie, quitte à le réintroduire ou à ré-augmenter sa posologie ensuite si nécessaire. Un IMC $\geq$ $30 \mathrm{~kg} / \mathrm{m}^{2}$ constitue un bon argument clinique pour le choix d'un GLP-1 RA. Le choix du liraglutide sera privilégié chez le patient en prévention cardiovasculaire secondaire (voir Avis $\left.n^{\circ} 26\right)$; sinon, il conviendra de privilégier le GLP-1 RA dont le coût journalier est le moins élevé ;

- une troisième possibilité est d'instaurer une insulinothérapie basale en association à la metformine (voir Avis $n^{\circ} 17$ pour la gestion des anti-hyperglycémiants lors de l'instauration d'une insuline basale).

- En cas d'hypoglycémies répétées ou sévères avec le sulfamide, une bithérapie alternative metformine + iDPP4 pourra également être envisagée.

\section{c. Après une bithérapie metformine + GLP-1 RA}

Avis $\mathrm{n}^{\circ} 12$ - Objectif d'HbA $\mathrm{Ac}_{1 \mathrm{c}}$ non atteint sous bithérapie metformine + GLP-1 RA (figure 4)

- Lorsque l'objectif d' $\mathrm{HbA}_{1 \mathrm{c}}$ n'est pas atteint sous bithérapie metformine + GLP-1 RA, il convient généralement d'instaurer une insulinothérapie basale. Dans ce cas, le GLP-1 RA sera conservé uniquement chez les patients ayant perdu du poids de façon cliniquement significative $(\geq 5 \%$ du poids initial) après introduction du GLP-1 RA. Une situation de prévention cardiovasculaire secondaire constitue un argument en faveur du maintien du liraglutide (voir Avis $n^{\circ} 26$ et Avis $n^{\circ} 17$ pour la gestion des anti-hyperglycémiants lors de l'instauration d'une insuline basale).

- Une deuxième option est de passer à une trithérapie metformine + GLP-1 RA + sulfamide.

- Enfin, dans le cas particulier d'une réponse thérapeutique insuffisante 3 ou 6 mois après initiation du GLP-1 RA (voir Avis $n^{\circ} 4$ ), une bithérapie metformine + sulfamide pourra également être envisagée, mais aucune étude n'a démontré l'efficacité de cette alternative.
5. Si $\mathrm{HbA}_{1 \mathrm{c}}>$ objectif personnalisé malgré modifications thérapeutiques du mode de vie + trithérapie à dose optimale bien observée (hors insuline)

Avis $\mathrm{n}^{\circ} 13$ - Objectif d'HbA $\mathrm{A}_{1 \mathrm{c}}$ non atteint sous trithérapie metformine + sulfamide + iDPP4 (figure 2 et figure 3 )

Lorsque l'objectif d' $\mathrm{HbA}_{1 \mathrm{c}}$ n'est pas atteint sous trithérapie metformine + sulfamide + iDPP4, le choix se fera entre :

- une association metformine + GLP-1 RA, en arrêtant toujours I'iDPP4 (sans objet avec un GLP-1 RA) et, le plus souvent, le sulfamide - quitte à le réintroduire secondairement si nécessaire ;

- l'instauration d'une insulinothérapie basale, en maintenant au moins la metformine (voir Avis $\mathrm{n}^{\circ} 17$ pour la gestion des anti-hyperglycémiants lors de l'instauration d'une insuline basale).

Avis $\mathrm{n}^{\circ} 14$ - Objectif d'HbA $\mathrm{H}_{1 \mathrm{c}}$ non atteint sous trithérapie metformine + GLP-1 RA + sulfamide hypoglycémiant (figure 4)

Lorsque l'objectif d'HbA ${ }_{1 c}$ 'est pas atteint sous trithérapie metformine + GLP-1 RA + sulfamide, il convient d'instaurer une insulinothérapie basale, en maintenant au moins la metformine (voir Avis $n^{\circ} 17$ pour la gestion des anti-hyperglycémiants lors de l'instauration d'une insuline basale).

\section{Initiation d'une insulinothérapie basale}

Avis ${ }^{\circ} 15$ - Initiation d'une insulinothérapie basale

- Lors du passage à l'insuline, il est recommandé de commencer par une injection quotidienne d'insuline basale.

- La mise en route d'une insulinothérapie basale nécessite une phase de préparation (co-décision avec le patient), ainsi qu'une éducation thérapeutique du patient (et de son entourage).

- On pourra commencer par une injection quotidienne avec de petites doses - par exemple 6 à $10 U$ par jour, à adapter en fonction du profil clinique du patient. II faudra mettre en place (ou renforcer) une auto-surveillance glycémique pour l'adaptation des doses d'insuline et la prévention des hypoglycémies. Dans la plupart des cas, pour obtenir une $\mathrm{HbA}_{1 \mathrm{c}}<7 \%$ (53 mmol/mol), il faudra viser une glycémie au réveil entre $0,80 \mathrm{~g} / \mathrm{L}$ et $1,30 \mathrm{~g} / \mathrm{L}$ et « titrer " l'insuline basale en ce sens (par exemple : adaptation des doses d'insuline tous les 3 jours en fonction des glycémies au réveil, la dose pouvant être augmentée ou réduite de 1 ou $2 \mathrm{U}$ ). 
- L'objectif est d'autonomiser le patient (et/ou son entourage) par une démarche d'éducation thérapeutique. L'intervention d'un(e) infirmier(ère) à domicile peut être parfois nécessaire, transitoirement ou durablement, pour certains patients.

- L'initiation de l'insuline basale peut quasiment toujours être proposée en ambulatoire.

- Un suivi rapproché devra être proposé pour vérifier la bonne réalisation, l'efficacité et la tolérance de l'insulinothérapie, modifier le protocole d'adaptation des doses si nécessaire, et adapter les traitements anti-hyperglycémiants associés.

- Le recours à un endocrinologue-diabétologue est souhaitable en cas de difficultés.

\section{Avis $\mathrm{n}^{\circ} 16$ - Choix de l'insuline basale}

- Lors de l'instauration d'une insuline basale, il est souhaitable de préférer un analogue lent de l'insuline à l'insuline neutral protamine Hagedorn (NPH), en raison d'un moindre risque hypoglycémique et d'une moindre variabilité glycémique.

- L'insuline glargine U100 est l'analogue lent de l'insuline dont la pharmacocinétique est la mieux adaptée à une majorité des patients. Pour des raisons économiques, il est souhaitable de privilégier un biosimilaire de la glargine.

- La pharmacocinétique des autres analogues lents de l'insuline (glargine U300, détémir) les rend utiles dans un certain nombre de situations individuelles, détaillées dans l'argumentaire (disponible sur www.sfdiabete.org). Le recours à un endocrinologue-diabétologue peut être utile dans ces cas-là.

Avis $n^{\circ} 17$ - Gestion des anti-hyperglycémiants lors de l'instauration d'une insuline basale

- Lors de l'instauration d'une insuline basale, il est souhaitable de :

$1 /$ maintenir la metformine ;

2/ arrêter le sulfamide (ou le répaglinide) d'emblée ou réduire sa posologie et l'arrêter après titration efficace de l'insuline basale, quitte à le réintroduire secondairement si nécessaire ; il convient de renforcer l'auto-surveillance glycémique pour ajuster la posologie du sulfamide (ou du répaglinide), et pour prévenir le risque hypoglycémique ;

3/ arrêter l'iDPP4 d'emblée ou après titration efficace de I'insuline basale, quitte à le réintroduire secondairement si nécessaire ;

4/ arrêter le GLP-1 RA sauf si celui-ci a permis une perte de poids cliniquement significative ( $\geq 5 \%$ du poids initial) ou s'il s'agit du liraglutide chez un patient en prévention cardiovasculaire secondaire (voir Avis $\mathrm{n}^{\circ} 26$ ).

- L'avis d'un endocrinologue-diabétologue est souhaitable en cas de difficultés.
7. Si $\mathrm{HbA}_{1 \mathrm{c}}>$ objectif personnalisé malgré modifications thérapeutiques du mode de vie + insuline basale bien titrée et bien observée

Avis $\mathrm{n}^{\circ} 18$ - Objectif d'HbA $\mathrm{Ac}_{1 \mathrm{c}}$ non atteint sous insulinothérapie basale + metformine (figure 5)

- En cas de résultats insuffisants sous insulinothérapie basale + metformine $\left(\mathrm{HbA}_{1 \mathrm{c}}>\right.$ objectif malgré des glycémies à jeun dans la cible, ou $\mathrm{HbA}_{1 \mathrm{c}}>$ objectif et glycémie à jeun au-dessus de la cible malgré de fortes doses d'insuline basale), l'avis d'un endocrinologue-diabétologue est souhaitable.

- À ce stade, l'ajout d'un autre traitement oral à l'insuline basale est une possibilité, mais elle est moins efficace que l'ajout d'un traitement injectable en termes de baisse $\mathrm{d}^{\prime} \mathrm{HbA}_{1 \mathrm{c}}$. Si cette option est choisie, il convient de privilégier l'ajout d'un iDPP4 plutôt que l'ajout d'un sulfamide ou du répaglinide pour limiter le risque hypoglycémique.

- Les deux autres options possibles sont :

- l'ajout d'un GLP-1 RA ;

- la mise en œuvre d'une insulinothérapie intensifiée par multi-injections (une ou plusieurs injections pré-prandiales d'un analogue rapide de l'insuline associées à une injection d'insuline basale, plutôt que 2 ou 3 injections quotidiennes d'insuline « premix ", schéma moins flexible pour le patient et généralement pourvoyeur de plus d'hypoglycémies et de prise de poids) ou, secondairement, dans des situations bien précises, et toujours après avis d'un endocrinologue-diabétologue, par pompe à insuline.

- Quelle que soit l'option choisie, et sauf apparition d'une contre-indication, il convient de conserver la metformine en association.

- Dans la majorité des cas, l'ajout d'un GLP-1 RA peut être proposé de préférence à une insulinothérapie intensifiée, en raison d'une efficacité au moins comparable, mais avec une plus grande simplicité et une meilleure tolérance (moins d'hypoglycémies, moins de prise de poids). De plus, une situation de prévention cardiovasculaire secondaire constitue un argument en faveur du liraglutide (voir Avis $n^{\circ} 26$ ). Le coût élevé de cette association thérapeutique doit amener à une réévaluation régulière de son effcacité et de sa tolérance.

- Une insulinothérapie intensifiée est parfois nécessaire d'emblée, en cas de déséquilibre glycémique majeur avec signes d'hyper-catabolisme ou de contre-indication ou d'intolérance aux GLP-1 RA. Cette insulinothérapie intensifiée sera aussi proposée secondairement, en cas d'échec d'une association insuline basale + GLP-1 RA. L'avis d'un endocrinologue-diabétologue est souhaitable dans ces situations complexes. 


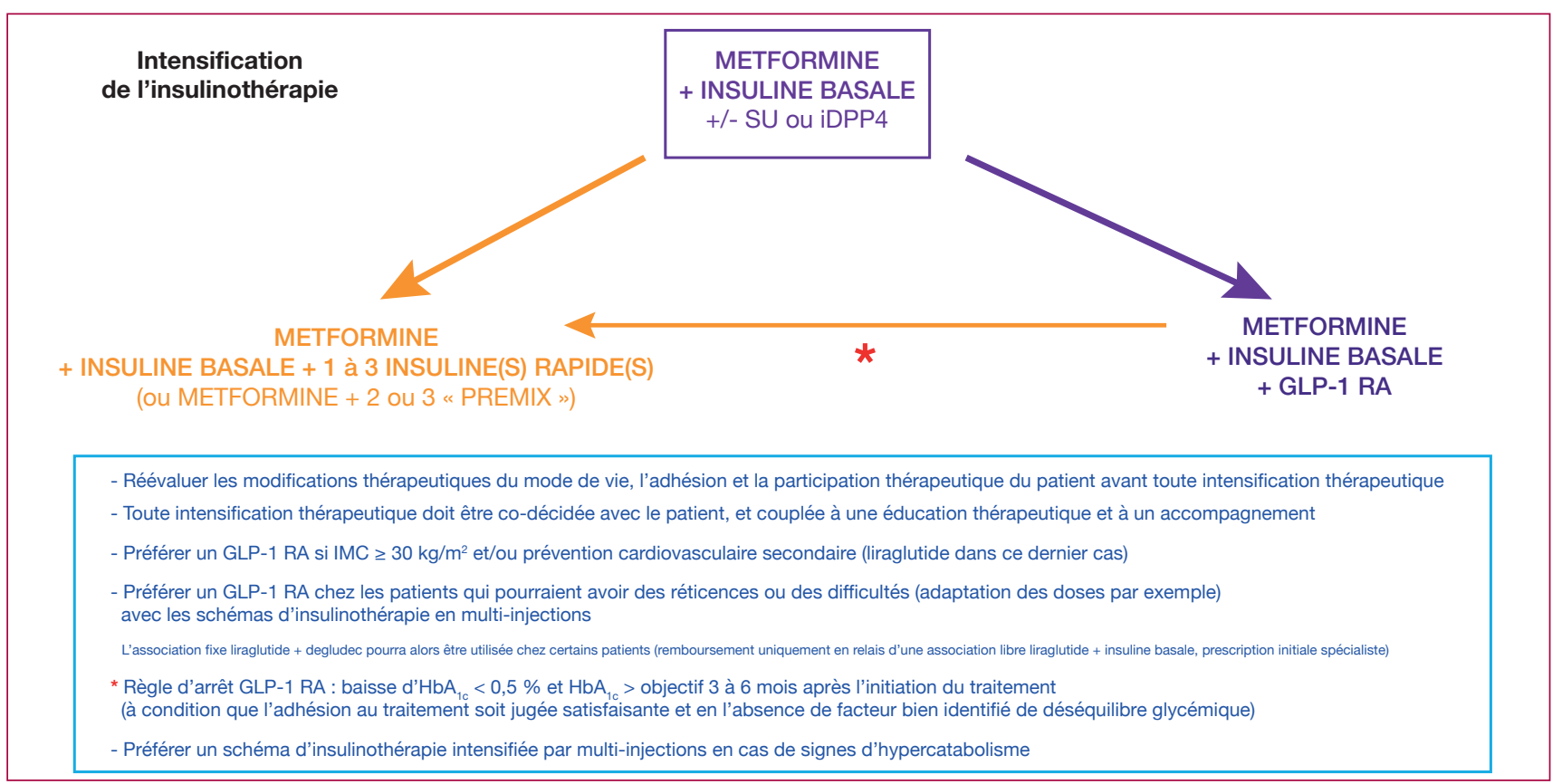

Figure 5. Stratégie thérapeutique si $\mathrm{HbA}_{1 \mathrm{c}}>$ objectif personnalisé malgré modifications thérapeutiques du mode de vie + insuline basale bien titrée bien observée.

\section{En cas d'intolérance avérée ou de contre-indication à la metformine}

Avis $n^{\circ} 19$ - En cas d'intolérance avérée ou de contre-indication à la metformine (figure 6)

- En cas de contre-indication ou d'intolérance digestive avérée à la metformine, on proposera un iDPP4 (non remboursé en France en monothérapie) ou un inhibiteur de l'alphaglucosidase (IAG) qui ont pour avantage de ne pas induire d'hypoglycémies ni de prise de poids ; un sulfamide ou le répaglinide, à faibles doses pour commencer, peuvent aussi être proposés, mais sont, pour leur part, associés à un risque d'hypoglycémie et de prise de poids.

- Lorsque l'objectif d' $\mathrm{HbA}_{1 \mathrm{c}}$ n'est pas atteint sous cette monothérapie initiale, et compte tenu du périmètre de remboursement des différentes classes thérapeutiques en France, on pourra proposer les associations suivantes : sulfamide + iDPP4 ou IAG ou GLP-1 RA ou insuline basale. - En troisième ligne, lorsque l'objectif d' $\mathrm{HbA}_{1 \mathrm{c}}$ n'est pas atteint sous bithérapie, il faudra recourir à une insulinothérapie exclusive.

\section{F. Populations particulières \\ 1. Patient âgé de plus de 75 ans}

Avis $\mathrm{n}^{\circ} 20$ - Patient âgé de plus de 75 ans : objectifs glycémiques (tableau I)

- Une évaluation gérontologique soigneuse doit être réalisée, prenant en compte différents paramètres : degré d'autonomie, conditions de vie, état cognitif, fonction rénale, co-morbidités, état nutritionnel, espérance de vie...

- Une attitude thérapeutique trop peu exigeante chez les patients âgés « en bonne santé » et un traitement trop intensif chez des sujets âgés " fragiles " sont les deux écueils à éviter.

- Les personnes âgées dites « en bonne santé ", autonomes d'un point de vue décisionnel et fonctionnel, et dont l'espérance de vie est jugée satisfaisante, peuvent bénéficier des mêmes cibles que les sujets plus jeunes ; pour les personnes âgées dites « fragiles ", une cible d' $\mathrm{HbA}_{1 \mathrm{c}}$ inférieure ou égale à $8 \%$ (64 mmol/mol) est recommandée; pour les personnes âgées dites " dépendantes et/ou à la santé très altérée ", la priorité est d'éviter les complications aiguës dues au diabète (déshydratation, coma hyperosmolaire), les complications infectieuses et les hypoglycémies ; des glycémies capillaires préprandiales comprises entre 1 et $2 \mathrm{~g} / \mathrm{L}$ et/ou un taux d'HbA ${ }_{1 c}$ inférieur à $9 \%(75 \mathrm{mmol} / \mathrm{mol})$ sont recommandés.

- De manière générale, chez les patients diabétiques âgés, il est essentiel de minimiser le risque d'hypoglycémie, notamment d'hypoglycémie sévère. Ce risque hypoglycémique existe sous sulfamides, répaglinide et insuline, et il est plus important, avec ces médicaments, lorsque le taux d' $\mathrm{HbA}_{1 \mathrm{c}}$ est inférieur à $7 \%$ (53 mmol $/ \mathrm{mol})$. 
Intolérence totale à la METFORMINE

\section{Monothérapie}

au choix
iDPP4 (AMM - non remboursé)

ou Acarbose

ou SU

ou Répaglinide

\begin{tabular}{|c|c|c|c|c|c|c|c|}
\hline $\begin{array}{l}\text { Bithérapie } \\
\text { au choix } \\
\text { (AMM) }\end{array}$ & $\begin{array}{l}\text { Acarbose } \\
+\mathrm{SU}\end{array}$ & ou & $\begin{array}{l}\text { iDPP4 } \\
+ \text { SU }\end{array}$ & ou & $\begin{array}{l}\text { GLP-1 RA } \\
+ \text { SU }\end{array}$ & ou & $\begin{array}{c}\text { INSULINE BASALE } \\
+/- \text { SU }\end{array}$ \\
\hline
\end{tabular}

au choix

+ SU

+ SU

$+\mathrm{SU}$

Insulinothérapie exclusive

Figure 6. Stratégies thérapeutiques en cas d'intolérance totale ou de contre-indication à la metformine.

Avis $\mathrm{n}^{\circ} 21$ - Patient âgé de plus de 75 ans : stratégie thérapeutique

- Plusieurs caractéristiques doivent être prises en compte dans la prise en charge du DT2 des personnes âgées : troubles cognitifs, altération de la fonction rénale, polymédication, dénutrition, risque élevé d'hypoglycémie et conséquences délétères de l'hypoglycémie...

- Les régimes restrictifs doivent être évités et une activité physique adaptée sera conseillée.

- La metformine reste le traitement de première ligne chez le sujet âgé, sous réserve du respect de ses contre-indications (notamment rénales, voir Avis $n^{\circ} 24$ ) et d'une vigilance accrue sur sa tolérance.

- Lorsque l'objectif d'HbA ${ }_{1 c}$ n'est pas atteint sous metformine, le choix se portera en priorité vers l'ajout d'un iDPP4.

- Lorsque l'objectif d'HbA $\mathrm{Ac}_{1 \mathrm{c}}$ 'est pas atteint sous association metformine + iDPP4 (voir Avis ${ }^{\circ} 10$ ), il convient de privilégier chez ces patients l'instauration d'une insuline basale. La prescription d'un sulfamide ou du répaglinide peut s'envisager chez certains sujets âgés "en bonne santé ", mais le risque hypoglycémique induit par ces produits est plus sérieux dans cette situation que dans d'autres. Après 75 ans, l'utilisation des GLP-1 RA doit être réservée à certains patients " en bonne santé ", idéalement après avis d'un endocrinologue-diabétologue, la recherche d'une perte de poids étant très rarement un objectif prioritaire à cet âge.

- Lorsque les autres traitements anti-hyperglycémiants ne peuvent pas être utilisés ou en cas de déséquilibre aigu (par exemple, épisode infectieux) ou chronique, l'insulinothérapie est recommandée, avec recours éventuel à une tierce personne et surveillance accrue des glycémies capillaires.

\section{Patient obèse avec IMC $\geq 35 \mathrm{~kg} / \mathrm{m}^{2}$}

Avis $\mathrm{n}^{\circ} 22$ - Patient obèse avec IMC $\geq 35 \mathrm{~kg} / \mathrm{m}^{2}$

- Chez le patient diabétique de type 2 obèse avec IMC $\geq 35 \mathrm{~kg} / \mathrm{m}^{2}$, après la metformine, on privilégiera les classes thérapeutiques neutres sur le plan pondéral (iDPP4, IAG) ou capables d'induire une perte de poids (GLP-1 RA).

- La chirurgie « métabolique " (sleeve gastrectomie, by-pass gastrique) constitue une option thérapeutique à considérer chez les patients diabétiques de type 2 de moins de 65 ans avec un IMC $\geq 40 \mathrm{~kg} / \mathrm{m}^{2}$, quelle que soit la qualité du contrôle glycémique, ou avec un IMC $\geq 35 \mathrm{~kg} / \mathrm{m}^{2}$, en cas de déséquilibre glycémique malgré un traitement anti-hyperglycémiant bien conduit. Le rapport bénéfices/risques de la chirurgie métabolique chez les patients diabétiques de type 2 présentant un IMC entre 30 et $35 \mathrm{~kg} / \mathrm{m}^{2}$, mal équilibrés malgré un traitement anti-hyperglycémiant bien conduit, est en cours d'évaluation ; à ce jour, cette indication n'est pas validée par la Haute Autorité de Santé (HAS).

- Dans tous les cas, l'approche chirurgicale ne doit être proposée qu'après une décision multidisciplinaire. Elle ne doit être réalisée que dans des centres ayant l'expertise nécessaire et requiert une surveillance post-opératoire régulière pour éviter la survenue d'éventuelles carences nutritionnelles et ajuster le traitement du diabète.

- Le traitement anti-hyperglycémiant doit souvent être allégé, et parfois supprimé, dans les suites immédiates de l'intervention, en particulier pour éviter des hypoglycémies, quitte à le reprendre secondairement en fonction de l'évolution des glycémies. 


\section{Patient présentant}

une insuffisance rénale chronique

Avis $n^{\circ} 23$ - Insuffisance rénale chronique (IRC) : objectifs glycémiques (tableau I)

- Chez les patients diabétiques de type 2 présentant une IRC modérée (DFG entre 30 et $60 \mathrm{~mL} / \mathrm{min} / 1,73 \mathrm{~m}^{2}$ ), on visera une $\mathrm{HbA}_{1 \mathrm{c}}$ cible inférieure ou égale à $7 \%(53 \mathrm{mmol} / \mathrm{mol})$.

- Chez les patients diabétiques de type 2 présentant une IRC sévère (DFG entre 15 et $30 \mathrm{~mL} / \mathrm{min} / 1,73 \mathrm{~m}^{2}$ ) ou terminale (DFG $<15 \mathrm{~mL} / \mathrm{min} / 1,73 \mathrm{~m}^{2}$ ), on visera une $\mathrm{HbA}_{1 \mathrm{c}}$ cible inférieure ou égale à $8 \%(64 \mathrm{mmol} / \mathrm{mol})$.

- Une coordination entre médecin généraliste, néphrologue et endocrinologue-diabétologue est recommandée, en particulier chez les patients avec un DFG $<45 \mathrm{~mL} / \mathrm{min} / 1,73 \mathrm{~m}^{2}$.
Avis $\mathrm{n}^{\circ} 24$ - Insuffisance rénale chronique (IRC) : gestion des anti-hyperglycémiants (tableau III)

- Au stade d'IRC modérée (DFG entre 30 et $60 \mathrm{~mL} / \mathrm{min} / 1,73$ m²), les molécules à élimination rénale doivent être utilisées avec précaution, car il existe un risque accru d'effets secondaires, notamment en ce qui concerne les hypoglycémies sous sulfamides ou insuline. La posologie de ces traitements sera adaptée, tout comme celle d'autres agents anti-hyperglycémiants, comme la metformine (dose maximale $1500 \mathrm{mg} / \mathrm{j}$ ), la vildagliptine (dose maximale $50 \mathrm{mg} / \mathrm{j}$ ) et la sitagliptine (dose maximale $50 \mathrm{mg} / \mathrm{j}$ ).

- Au stade d'IRC sévère (DFG : 15 à $30 \mathrm{~mL} / \mathrm{min} / 1,73 \mathrm{~m}$ ), seuls l'insuline, le répaglinide (avec un risque d'hypoglycémies pour ces deux traitements), le liraglutide, et la vildagliptine à la dose de $50 \mathrm{mg} / \mathrm{jour}$ peuvent être utilisés.

- Au stade d'IRC terminale (DFG $<15 \mathrm{~mL} / \mathrm{min} / 1,73 \mathrm{~m}^{2}$ ), seuls l'insuline, le répaglinide (avec un risque d'hypoglycémies pour ces deux traitements) et la vildagliptine à la dose de $50 \mathrm{mg} / \mathrm{jour}$ peuvent être utilisés.

\begin{tabular}{|l|l|l|l|l|}
\hline $\begin{array}{l}\text { DFG } \\
\left(\mathrm{mL} / \mathrm{min} / 1,73 \mathrm{~m}^{2}\right)\end{array}$ & $\begin{array}{l}\text { IRC légère } \\
>59 \text { à } \leq 89\end{array}$ & $\begin{array}{l}\text { IRC modérée } \\
\geq 30 \text { à } \leq 59\end{array}$ & $\begin{array}{l}\text { IRC sévère } \\
<30\end{array}$ & $\begin{array}{l}\text { IRC terminale } \\
\text { Dialyse }\end{array}$ \\
\hline Insuline & & & & \\
\hline Exenatide & & & & \\
\hline Exenatide retard & & & & \\
\hline Dulaglutide & & & & \\
\hline Liraglutide & & & & \\
\hline Vildagliptine & & & & \\
\hline Sitagliptine & & & & \\
\hline Saxagliptine & & & & \\
\hline Metformine & & & & \\
\hline Acarbose & & & & \\
\hline Répaglinide & & & & \\
\hline Glimépiride & & & \\
\hline Gliclazide & & & \\
\hline \\
\hline
\end{tabular}

Tableau III. Fonction rénale (DFG estimé) et utilisation des anti-hyperglycémiants. 


\section{Patient en prévention cardiovasculaire secondaire}

Avis n`25 - Patient en prévention cardiovasculaire secondaire : objectifs glycémiques (tableau I)

- Chez les patients diabétiques de type 2 avec un antécédent de pathologie cardiovasculaire considérée comme non évoluée, on visera une $\mathrm{HbA}_{1 \mathrm{c}}$ cible inférieure ou égale à $7 \%$ (53 mmol/mol).

- Chez les patients diabétiques de type 2 avec un antécédent de pathologie cardiovasculaire considérée comme évoluée*, on visera une $\mathrm{HbA}_{1 \mathrm{c}}$ cible inférieure ou égale à $8 \%(64 \mathrm{mmol} / \mathrm{mol})$.

- Une coordination entre médecin généraliste, cardiologue et endocrinologue-diabétologue est recommandée.

* Antécédent de pathologie cardiovasculaire considérée comme évoluée : IDM avec insuffisance cardiaque, atteinte coronarienne sévère (atteinte du tronc commun ou atteinte tritronculaire ou atteinte de l'artère interventriculaire antérieure proximale), atteinte polyartérielle (au moins deux territoires artériels symptomatiques), artériopathie oblitérante des membres inférieurs symptomatique, accident vasculaire cérébral récent $(<6$ mois).

Avis $n^{\circ} 26$ - Patient en prévention cardiovasculaire secondaire : stratégie thérapeutique

- La stratégie " commune » de prise en charge du DT2 peut être suivie chez les patients en prévention cardiovasculaire secondaire en portant une attention particulière au risque d'hypoglycémie.

- La metformine doit être maintenue ou introduite sous réserve du respect des contre-indications.

- Pour les patients en prévention cardiovasculaire secondaire, dont le phénotype clinique et biologique justifie la prescription d'un traitement par GLP-1 RA, le liraglutide doit être privilégié, en raison de son bénéfice cardiovasculaire démontré dans l'étude LEADER dans cette situation.

- Dans les cas où une insulinothérapie basale est envisagée chez un patient recevant déjà un GLP-1 RA, le maintien du liraglutide doit être envisagé chez les patients en prévention cardiovasculaire secondaire.

- Chez un patient en prévention cardiovasculaire secondaire, si le choix en deuxième ligne s'oriente vers un traitement oral ou s'il apparaît que le liraglutide n'est pas souhaitable (mauvaise tolérance aux GLP-1 RA, sujet âgé...), le recours à la sitagliptine est à privilégier compte tenu de sa sécurité d'emploi démontrée dans l'étude TECOS (pour Trial Evaluating Cardiovascular Outcomes with Sitagliptin) chez les patients en prévention secondaire, sans sur-risque d'hospitalisation pour insuffisance cardiaque.

\section{Patient présentant une insuffisance cardiaque}

Avis $n^{\circ} 27$ - Patient présentant une insuffisance cardiaque

Chez un patient diabétique de type 2 présentant une insuffisance cardiaque :

1/ la metformine doit être le traitement de première intention s'il s'agit d'une insuffisance cardiaque stable sans IRC sévère associée (DFG $>30 \mathrm{~mL} / \mathrm{min} / 1,73 \mathrm{~m}^{2}$ ), mais doit être évitée en cas d'insuffisance cardiaque instable et/ou nécessitant une hospitalisation ;

2/ la sitagliptine a démontré, dans l'étude TECOS, sa sécurité vis-à-vis du risque de mortalité et/ou d'hospitalisation liés à l'insuffisance cardiaque, alors que la saxagliptine augmente le risque d'hospitalisation pour insuffisance cardiaque dans l'étude SAVOR-TIMI 53 (pour Saxagliptin Assessment of Vascular Outcomes Recorded in patients with diabetes mellitus-Thrombolysis in Myocardial Infarction 53) et doit donc être évitée ;

3/ toutes les autres classes d'anti-hyperglycémiants disponibles sur le marché peuvent être utilisées, mais aucun médicament n'a montré, à ce jour, de bénéfice particulier sur les événements liés à l'insuffisance cardiaque, à l'exception de l'empagliflozine et de la canagliflozine, inhibiteurs du co-transporteur sodium/glucose de type 2 (iSGLT2) non commercialisés en France actuellement ;

4/ les sulfamides et le répaglinide ne seront pas privilégiés, en raison du risque hypoglycémique ;

5/ les GLP1-RA doivent être évités en cas d'insuffisance cardiaque à fraction d'éjection diminuée, compte tenu de l'accélération du rythme cardiaque et du signal défavorable observé avec le liraglutide dans les études LIVE (pour effect of LIraglutide on left VEntricular function in chronic heart failure patients with and without type 2 diabetes mellitus) et FIGHT (pour Functional Impact of GLP-1 for Heart failure Treatment) chez de tels patients ;

6/ une coordination entre généraliste, cardiologue et endocrinologue-diabétologue est recommandée. 


\section{Patiente enceinte ou envisageant de l'être}

Avis $n^{\circ} 28$ - Patiente enceinte ou envisageant de l'être (texte repris des recommandations HAS/ansm 2013)

«Les femmes en âge de procréer doivent être informées de l'intérêt d'un bon contrôle glycémique avant et durant la grossesse afin d'améliorer le pronostic obstétrical. Une cible d' $\mathrm{Hb} A_{1 c}$ si possible inférieure à $6,5 \%$ est recommandée avant d'envisager la grossesse. Durant la grossesse, l'équilibre glycémique doit être évalué par des glycémies capillaires pluriquotidiennes. Des glycémies inférieures à $0,95 \mathrm{~g} / \mathrm{L}$ à jeun et $1,20 \mathrm{~g} / \mathrm{L}$ en postprandial à 2 heures et un taux d'HbA ${ }_{1 c}$ inférieur à $6,5 \%$, sous réserve d'être atteints sans hypoglycémie sévère, sont recommandés.

Les risques de complications maternelles et fœtales peuvent être réduits en optimisant le contrôle glycémique avant le début et tout au long de la grossesse. Idéalement, la femme enceinte diabétique doit être prise en charge avant la conception. Du fait de l'importance d'un contrôle glycémique optimal dès le début de la grossesse, les anti-diabétiques oraux non foeto-toxiques* ne doivent pas être interrompus avant la mise sous insuline et celle-ci doit être la plus précoce possible. Le schéma optimisé fait souvent appel à des schémas multiinjections comprenant des analogues de l'insuline rapide et l'insuline lente ou intermédiaire ${ }^{* *}$. Les besoins nutritionnels de la femme enceinte diabétique sont les mêmes que pour la femme enceinte non diabétique.

Il est recommandé de mettre en place un schéma insulinique optimisé le plus précocement possible, afin d'atteindre et de respecter les objectifs glycémiques stricts. Une coordination étroite entre équipe gynéco-obstétricale, équipe endocrinologique et médecin généraliste est recommandée. Cette prise en charge doit débuter avant la conception. ”

* à savoir la metformine ("Les données publiées chez les femmes enceintes exposées à la metformine au $1^{\text {er }}$ trimestre sont très nombreuses et rassurantes ") et le glibenclamide ("Les données publiées chez les femmes enceintes exposées au glibenclamide au $1^{\text {er }}$ trimestre sont peu nombreuses, mais aucun effet malformatif n'est retenu à ce jour ") [Source : Centre de Référence sur les Agents Tératogènes (CRAT), Hôpital Armand-Trousseau, Paris ; https://lecrat.fr/]

** Les insulines disposant d'une AMM pendant la grossesse sont l'insuline rapide humaine, la NPH, et l'aspart ; les données sur un grand nombre de grossesses exposées n'indiquent pas d'effet indésirable de l'insuline lispro, de la détémir et de la glargine U100 sur la grossesse ou sur la santé du foetus/nouveau-né, et la prescription de ces insulines peut être envisagée pendant la grossesse si nécessaire.

\section{G. Place de l'auto-surveillance glycémique chez le patient diabétique de type 2}

Avis $\mathrm{n}^{\circ} 29$ - Auto-surveillance glycémique

chez le patient diabétique de type 2 (texte

repris des recommandations HAS/ansm 2013)

"L'auto-surveillance glycémique (ASG) doit s'inscrire dans une démarche d'éducation du patient. Lors de la prescription d'un dispositif d'ASG, il est indispensable d'expliquer au patient et d'organiser avec lui cette auto-surveillance, de définir les moments, la fréquence, les objectifs et les décisions à prendre en fonction des résultats. Les résultats recueillis serviront de support de discussion entre le patient et l'équipe soignante.

L'ASG est recommandée chez les patients diabétiques de type 2 traités par insuline, afin d'adapter les doses d'insuline et de prévenir les hypoglycémies.

L'ASG est recommandée pour les patientes enceintes ou envisageant de l'être.

L'ASG peut être utile chez les patients diabétiques de type 2 traités par sulfamides ou glinides afin de prévenir et de détecter d'éventuelles hypoglycémies.

L'ASG peut être utile :

- pour évaluer l'effet de modifications du style de vie ou des traitements;

- en cas de risque de déséquilibre aigu (infections, corticothérapie, interruption de traitement antidiabétique, etc.);

- pour les patients avec un taux d' $H b A_{1 c}>8 \%$ dans le cadre d'un ajustement thérapeutique dont le passage à l'insuline ;

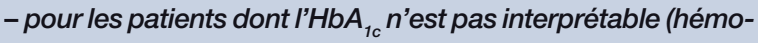
lyse, etc.)

L'ASG n'est recommandée que si les résultats sont susceptibles d'entraîner une modification des mesures hygiéno-diététiques et/ou du traitement médicamenteux. La réalisation systématique de l'ASG, chez les patients sous antidiabétiques ne provoquant pas d'hypoglycémie, n'est pas recommandée. » 


\section{H. Dimension économique}

\section{Avis $\mathrm{n}^{\circ} 30$ - Dimension économique}

- La prise en compte des conséquences économiques collectives dans les recommandations en matière de traitements du DT2 est légitime, et ce, d'autant plus que cette pathologie chronique a une prévalence croissante. La règle éthique « du juste soin pour le patient au moindre coût pour la collectivité " nécessite une amélioration de la pertinence des actes et des prescriptions, ainsi que la recherche de l'efficience dans les organisations de soins.

- Les médicaments génériques et biosimilaires, moins onéreux, doivent être privilégiés.

- En dehors du cas particulier des génériques et des biosimilaires, la dimension économique ne doit être prise en compte dans le choix d'un médicament que lorsque celui-ci présente un rapport bénéfices/risques au moins équivalent à celui du traitement de comparaison. En outre, au-delà du prix des produits, il convient de considérer le coût global induit par le traitement (par exemple : renforcement de l'ASG, survenue d'hypoglycémies, recours à un(e) infirmier(ère) à domicile...). Idéalement, il faudrait rapporter l'ensemble des coûts induits par les traitements à leur efficacité. Peu d'études ont été conduites en ce sens sur les traitements du diabète en France et la validité des études publiées est incertaine.

- L'efficacité des iDPP4 et des GLP-1 RA devra être soigneusement réévaluée à intervalles réguliers, compte tenu de leur prix supérieur à celui des sulfamides.

- Il importe, dans le futur, de mettre en place les conditions permettant une évaluation économique systématique des stratégies thérapeutiques dans la prise en charge du DT2 et d'inciter à la conduite d'études en ce sens. 